\title{
Imaging genomics
}

\author{
Paul M. Thompsona ${ }^{\mathrm{a}}$, Nicholas G. Martin ${ }^{\mathrm{b}}$, and Margaret J. Wright ${ }^{\mathrm{b}}$ \\ a Laboratory of Neuro Imaging, Department of Neurology, UCLA School of Medicine, Los \\ Angeles, California, USA \\ ${ }^{b}$ Queensland Institute of Medical Research, Brisbane, Australia
}

\begin{abstract}
Purpose of review-Imaging genomics is an emerging field that is rapidly identifying genes that influence the brain, cognition, and risk for disease. Worldwide, thousands of individuals are being scanned with high-throughput genotyping (genome-wide scans), and new imaging techniques [high angular resolution diffusion imaging and resting state functional magnetic resonance imaging (MRI)] that provide fine-grained measures of the brain's structural and functional connectivity. Along with clinical diagnosis and cognitive testing, brain imaging offers highly reproducible measures that can be subjected to genetic analysis.
\end{abstract}

Recent findings-Recent studies of twin, pedigree, and population-based datasets have discovered several candidate genes that consistently show small to moderate effects on brain measures. Many studies measure single phenotypes from the images, such as hippocampal volume, but voxel-wise genomic methods can plot the profile of genetic association at each 3D point in the brain. This exploits the full arsenal of imaging statistics to discover and replicate gene effects.

Summary-Imaging genomics efforts worldwide are now working together to discover and replicate many promising leads. By studying brain phenotypes closer to causative gene action, larger gene effects are detectable with realistic sample sizes obtainable from meta-analysis of smaller studies. Imaging genomics has broad applications to dementia, mental illness, and public health.

\section{Keywords}

diffusion imaging; genome-wide association study; heritability; MRI; twins

\section{Introduction}

Imaging genetics is a rapidly emerging field that is opening up a new landscape of discovery in medicine and neuroscience. The field is a hybrid effort that merges methods and discoveries in both imaging and genetics; its power has recently taken a quantum leap for a number of reasons. First, many groups worldwide are scanning thousands of individuals with structural and functional magnetic resonance imaging (MRI). Samples are now large enough to discover and verify effects of specific genes on the brain [1-3]. Second, voxelwise genomic methods are emerging that search every location in a brain image for statistical effects of genes $\left[4^{*}\right]$. These approaches identify coherent anatomical patterns of

Correspondence to Dr Paul Thompson, Laboratory of Neuro Imaging, Dept. of Neurology, UCLA School of Medicine, 635 Charles E. Young Drive South, Suite 225E, Los Angeles, CA 90095-7332, USA Tel: +1 310206 2101; fax: +1 3102065518 ;

thompson@loni.ucla.edu.

The authors report no conflicts of interest, financial or otherwise, regarding the work in this study. 
gene effects in 3D. Replication efforts can then focus on selected brain measures that show promise in preliminary analyses.

Finally, several imaging measures that may seem too unstable to be measured reproducibly have been shown to be highly heritable and feasible to collect in large numbers of individuals. Recent functional MRI studies in healthy adults found that patterns of taskrelated brain activation $[5,6]$ and resting state functional connectivity $\left[7,8^{\circ}\right]$ are heritable and highly reproducible, making it more likely to discover genes that influence these traits. MRI variants such as diffusion tensor imaging (DTI) -which is sensitive to the directional diffusion of water along neural pathways - can be used to identify entirely new measures of brain integrity and connectivity [9]. By scanning large cohorts of twins with high angular resolution diffusion imaging (HARDI), Chiang et al. $\left[10^{\circ}\right]$ revealed that the fiber integrity of the brain is under strong genetic control, and is highly correlated with cognitive measures such as intelligence quotient. Bivariate genetic analysis can also be used to show that common sets of underlying genes affect cognition and fiber integrity [10 $0^{\circ}$ or cognition and regional brain volumes $[11,12]$.

\section{How can gene effects be studied?}

Current efforts to relate genetic variation to imaging data typically proceed in one of several directions. The most direct method is to study an illness with a genetic basis, such as fragile $\mathrm{X}$ syndrome, Turner syndrome, Williams syndrome, or 22q11.2 deletion syndrome [13]. Neuroimaging of groups of patients with neurodevelopmental disorders has been extremely useful in discovering the brain systems affected, when and where in the brain abnormalities emerge, and where identifiable abnormalities of brain structure and function fit on the pathway from molecular dysfunction to behavior [14]. Fragile X syndrome, for example, involves a genetic abnormality in the expression of a protein, FMRP, which is involved in dendritic pruning [15]. As such, blood levels of the protein have been related to identifiable patterns of brain hypertrophy in the caudate nucleus, when the normal pruning process is derailed [16]. Brain mapping studies of Williams syndrome have also revealed regionally altered cortical complexity [17], cortical folding [18], and diffusion anisotropy [19] in characteristic patterns that may be related to the expression of genes in the deleted region of the genome. Some of these genes, such as elastin, are involved in cortical folding. In many of these studies, imaging provides a quantifiable phenotype in the brain that can be monitored over time, leading to a more mechanistic understanding of behavioral abnormalities in neurogenetic disorders, and a means to assess their onset and progression.

A second approach to imaging genetics is to fit quantitative genetic models to data from twins or other related individuals [20]. This can identify features or traits that are genetically influenced. Even the most associated common genetic polymorphisms are individually expected to explain less than $1-5 \%$ of the variation in even the most heritable brain measures, so the first step in an analysis is often to show that the brain measures of interest are genetically influenced at all. Twin studies, in particular, reveal that many aspects of

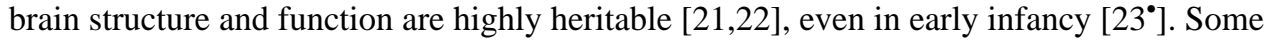
studies are also beginning to identify common genetic underpinnings for different brain measures and different brain regions [24]. The classical twin design examines correlations between identical and fraternal twins, and compares the observed data with what would be predicted under different types of genetic models. Bivariate modeling can also reveal how correlations among brain phenotypes and cognitive measures are underpinned by the same sets of genes [21]. Curiously, different measures of the same structure - such as cortical thickness and cortical surface area - can both be highly heritable but determined by different sets of genes [24-26]. Conversely, the diffuse effects of single genes on the brain, such as brain-derived neurotrophic factor $(B D N F)$, are not necessarily localized, and may be 
detected across many structures at once, due to the observed covariance among brain volumes [27-29]. These genetic modeling efforts have been greatly accelerated by software packages for efficient computational analysis, such as Mx [30] and SOLAR [31] - which now has 4000 registered users, and has spurred the widespread use of quantitative genetic analysis.

\section{Statistical brain maps}

Early studies focused on single measures derived from an MRI scan - such as the total volume of the brain, or the total amount of gray or white matter in each lobe. Even so, in the last decade the brain imaging field has seen increasingly widespread use of statistical brain mapping methods. Statistical parametric maps, or SPMs, are now widely used to plot profiles of statistical effects on the brain in 3D. These include effects on brain morphometry and functional activation. In this approach, automated methods are first used to align brain images from hundreds of individuals to a common digital image template. In the aligned datasets, regression models may be used to detect profiles of brain signals found consistently in a population, and factors that influence them. Thompson et al. [32] used this type of voxel-based analysis to make 3D maps of the heritability of cortical gray matter volumes, across the cortical surface in 3D. This twin study was the first to show the pattern of heritability and other genetic statistics at each point in the brain, in the form of a 3D map. This and subsequent analyses showed that cortical gray matter volumes, especially in the frontal lobes, were highly heritable, consistent with reports of gray matter deficits in relatives of unaffected patients with schizophrenia [33]. Hulshoff Pol et al. [34] used an image warping method to deform over 100 twins' MRI scans to a common brain template to estimate the volumes of different parts of the brain. Most structure volumes were highly heritable; other structures, such as the cerebellum, were more environmentally influenced. Statistical mapping methods may also be used to transfer other types of imaging data to a common template, and perform voxel-by-voxel genetic analysis. In one morpho-metric study of twins, the degree of genetic control was found to be higher for earlier-maturing regions of the brain, such as the visual processing areas of the occipital lobes. In line with expectation, frontal lobe white matter structures with a more protracted maturational timecourse were more environmentally influenced [35]. Paradoxically, however, there is some evidence for an increasing genetic effect on cortical thickness with age [36], in agreement, perhaps, with the rising heritability of intelligence quotient with age [37,38]. Importantly, these genetic mapping studies find anatomical and time-dependent gradients in the pattern of genetic influences. Mapping methods can then home in on selected regions of the brain in which genetic effects were most likely to be detected. This type of voxel-selection approach -when candidate brain regions are prescreened for future study - is the mainstay of functional brain imaging, and avoids the need for heavy corrections for multiple statistical comparisons that occur when surveying the entire brain.

\section{Candidate genes}

Given the enormous number of possible genes that could influence the brain, candidate genes offer a more principled method to focus on promising regions of the genome. Perhaps the most studied polymorphism in brain imaging is the apolipoprotein E4 (ApoE4) allele, which is carried by approximately $25 \%$ of normal individuals; each allele confers a threefold increased risk for developing late-onset Alzheimer's disease by 75 years of age. ApoE4 is now widely recognized as leading to reduced gray matter $[39,40]$ and white matter [41] volumes in the elderly, perhaps due to the toxic effects of beta-amyloid burden, which is also higher in ApoE4 carriers [42,43]. Somewhat disturbingly, Shaw et al. [44] found that children carrying this common gene also showed a pattern of cortical thinning and an altered developmental trajectory; resting-state brain activity is also altered in young ApoE4 carriers 
(age 20-35; [45]). In one of a series of studies associating BDNF variants with alterations in brain structure, activation, and cognition (e.g. [46]), Chiang et al. [47] discovered that a common polymorphism in the $B D N F$ gene influences the fiber integrity of the brain, as seen with DTI. The $B D N F$ polymorphism accounted for around $15 \%$ of the variance in diffusion anisotropy in the posterior outflow of the corpus callosum [47], but even so, scans from 455 twins were required to detect and replicate this association, suggesting the need for large imaging databases.

Genes coding for common variants in monoamine neurotransmitter receptors and transporters are a common target of study [e.g. [48-51] on catechol- $O$-methyl transferase $(C O M T)$; [52] on the dopamine transporter, DAT1; and [53] relating variations in the dopamine D2 receptor to functional connectivity]. Hariri et al. [54] and others have reported that people carrying one or two copies of the short allele of the serotonin transporter (5$H T T$ ) promoter polymorphism, which has been associated with reduced 5-HTT expression and function and increased fear and anxiety-related behaviors, exhibit greater neuronal activity in the amygdala, as assessed by BOLD functional MRI, in response to fearful stimuli. Other candidate gene discoveries are relevant to public health. Ho et al. [55 $5^{\circ}$ found that FTO, an obesity-related gene variant carried by nearly half of all Western Europeans, may also be associated with brain degeneration. In 3D maps based on over 200 healthy elderly Caucasian individuals' MRI scans, carriers' brains demonstrated $8 \%$ lower volume than noncarriers in the frontal lobes and $12 \%$ lower volume in the occipital lobes. FTO may exert an additive effect on brain degeneration beyond the influence of an individual's body mass index, which is also associated with atrophy and differences in brain structure [56].

\section{Genome-wide association studies}

Candidate gene studies can provide a better understanding of the pathways involved in several brain diseases, but the advent of high-throughput genotyping has offered still greater potential to search the entire genome for causative variants. Genome-wide association (GWA) scanning, in particular, searches the entire genome for single-nucleotide polymorphisms (SNPs) that may be associated with certain behaviors, diseases, or, in the case of imaging genomics, with imaging measures. Figure 1 shows how such an approach works (adapted from [57]).

In this preliminary GWA study of neurodegeneration, Stein et al. [57] searched 546314 genomic markers using the PLINK software [58]. Two SNPs were suggestively associated with temporal lobe volumes $\left(P<5 \times 10^{-7}\right)$, and with increased atrophy in all three diagnostic categories [Alzheimer's disease, mild cognitive impairment (MCI), and controls]. One SNP, with genome-wide evidence, was in the GRIN2B gene that encodes the $N$-methyl- $D$ aspartate (NMDA) glutamate receptor NR2B subunit. It was also over-represented in Alzheimer's disease and MCI patients versus controls [odds ratio $(\mathrm{OR})=1.273 ; P=0.039$ ]. This protein, involved in learning and memory and excitotoxic cell death, has age-dependent prevalence in the synapse, and is already a therapeutic target in Alzheimer's disease [59]. Voxel-by-voxel, 3D maps of genetic association with regional brain volumes revealed intense temporal lobe reductions of around $1.5 \%$ per risk allele (Fig. 1).

\section{Rare variants}

Dickson et al. $\left[60^{\circ}\right]$ have proposed, somewhat provocatively, that uncommon or rare genetic variants can easily create synthetic associations that are credited to common variants, and this should be considered when interpreting GWA scanning signals. Conventional GWA scans may also miss a large component of causal variance due to rare functional variation. With this in mind, Choi et al. [61] and others have advocated finer-scale exome sequencing of pedigrees, to identify all polymorphisms that might be relevant to a brain phenotype. As 
sequencing becomes less expensive, the relative contribution of rare versus common variation to brain phenotypes is likely to be better understood. Imputation to the 1000 Genomes Project (up to 8 million SNPs) and the advent of much denser SNP chips will provide cheaper options in the short term.

\section{Voxel-wise genomics: statistical and computational challenges}

Most genome-wide studies of brain images reduce the dimension of one or both datasets to avoid performing statistical tests on an astronomical scale. Meda et al. [62], for example, advocated independent component analysis to identify the association between sets of genomic variations and phenotypic traits, identifying interactions between patterns of brain function and genetic information. Other multivariate methods, such as canonical correlation analysis, have been used to find optimal 'projections' to best correlate the multivariate measures of genotypes and phenotypes [63].

Stein et al. [4•], however, used a massively parallel computer to associate approximately 600000 SNPs at approximately 200000 voxels in the brain, requiring approximately $1.2 \times$ $10^{11}$ tests on data from 742 individuals. To address the problem of multiple comparisons across both the genome and the image, only the 'winning' (most highly associated) SNP was retained at each voxel, and its $P$ value was plotted into the image. A beta function was fitted to model the distribution of minimum $P$ values that would be obtained under the null hypothesis. False discovery rate methods [64] were used to adjust for multiple tests across the image. Although the list of promising genes requires replication, clearly if several centers perform such an analysis, the resulting maps can be combined in several simple ways. Statistical conjunction maps [65], for example, could identify subsets of voxels and SNPs with weak or strong evidence of association in multiple datasets. Clearly, genes are more likely to operate in networks, and interactions are likely; machine learning methods, such as adaptive boosting have been proposed to identify sets of SNPs with large combined effects that would be hard to detect independently. Recognizing that individual variants are likely to have small effect, risk profiling has also been proposed [66] to identify sets of SNPs that jointly explain the observed variance. Genes may also interact [e.g. BDNF and SLC6A4 (5-HTTLPR)], or the dopamine receptor and transporter [67], and tools to detect these interactions are just beginning to be developed $[68,69]$.

\section{Replication by joining datasets}

A major barrier in imaging genomics is replication; without it, GWA findings are met with skepticism [70]. Recently, Chiang et al. [65] discovered, and replicated, a finding that a nonsynonymous coding variant in the $B D N F$ gene affects white matter microstructure and its relation to intelligence quotient. In parallel work, Kochunov et al. [3] performed GWA on a large diffusion imaging dataset from a Mexican-American pedigree, and revealed a list of promising genes that affect white matter circuitry and brain structure. Given the large samples needed to discover and verify promising hits, several imaging genomics groups are now working collaboratively to replicate findings $\left[71^{\circ}\right]$. The ENIGMA network (Enhancing Neuroimaging Genetics through Meta-Analysis; http://enigma.loni.ucla.edu) seeks to accelerate replication by sharing information on promising findings, and identifying cohorts with sufficient power for meta-analysis. This approach has been fruitful in psychiatry and behavioral genetics $\left[72^{\circ *}\right]$.

\section{Conclusion}

Imaging genetics is an emerging field evaluating imaging measures as quantitative traits. Many imaging phenotypes have high precision, and are readily standardized across centers [73]. They may also require smaller sample sizes to detect association [74] - and arguably, 
they may be closer to the underlying biology of disease than behavioral or standard diagnostic measures, making contributing genes easier to identify.

The flurry of recent discoveries in imaging genomics underscores the fact that imaging datasets are now large enough to discover single gene effects on brain structure and function. Several candidate genes show reproducible effects on the brain and risk for disease; long lists of new candidates have been discovered by mining genetic data. Replication is key and collaborations such as the ENIGMA network have been set up to replicate promising findings across multiple independently collected samples. In parallel, several groups have extended genomic analysis to new types of images: diffusion-based maps of fiber integrity and connectivity [9], and 3D maps of task-related brain activation and 'resting-state' functional connectivity $\left[5,8^{\circ}\right]$. Together, these advances are spurring many new discoveries, providing valuable mechanistic information about the brain and behavior, and about factors that affect the expression of neurological and psychiatric illnesses.

\section{Acknowledgments}

The authors are supported by NIH grant number RO1 HD050735 from the National Institute of Child Health and Human Development, USA, and Project Grant 496682 from the National Health and Medical Research Council, Australia. P.T. is also supported, in part, by NIH grants EB008432, EB008281, EB007813, AG036535, RR021813 and RR013642.

\section{References and recommended reading}

Papers of particular interest, published within the annual period of review, have been highlighted as:

- of special interest

•• of outstanding interest

Additional references related to this topic can also be found in the Current World Literature section in this issue (pp. 434-435).

1. Potkin SG, Guffanti G, Lakatos A, et al. Hippocampal atrophy as a quantitative trait in a genomewide association study identifying novel susceptibility genes for Alzheimer's disease. PLoS One 2009;4:e6501. [PubMed: 19668339]

2. Shen L, Kim S, Risacher SL, et al. Whole genome association study of brain-wide imaging phenotypes for identifying quantitative trait loci in MCI and AD: a study of the ADNI cohort. Neuroimage. 2010 [Epub ahead of print].

3. Kochunov P, Glahn DC, Lancaster J, et al. Genetics of microstructure of cerebral white matter using diffusion tensor imaging. NeuroImage. 2010 [Epub ahead of print].

4•. Stein JL, Hua X, Lee S, et al. Voxelwise genome-wide association study (vGWAS). NeuroImage. 2010 [Epub ahead of print]. This voxel-wise genomics study was the first to test for genetic associations at all voxels in a brain image, and across all locations on the genome, leading to a new approach to screen image databases for genetic effects.

5. Blokland GAM, McMahon KL, Hoffman J, et al. Quantifying the heritability of task-related brain activation and performance during the N-back working memory task: a twin fMRI study. Biol Psychiatry 2008;79:70-79.

6. Koten JW Jr, Wood G, Hagoort P, et al. Genetic contribution to variation in cognitive function: an fMRI study in twins. Science 2009;323:1737-1740. [PubMed: 19325117]

7. Esslinger C, Walter H, Kirsch P, et al. Neural mechanisms of a genome-wide supported psychosis variant. Science 2009;324:605. [PubMed: 19407193] 
8•. Glahn DC, Winkler AM, Kochunov P, et al. Genetic control over the resting brain. Proc Natl Acad Sci U S A 2010;107:1223-1228. This study examines the so-called default network - a set of brain regions whose activity is correlated even when a individual lies at rest in the scanner. The properties of this network were shown to be heritable, paving the way for imaging genomic studies of functional connectivity in the brain. [PubMed: 20133824]

9. Patel, V.; Chiang, MC.; Thompson, PM., et al. Scalar connectivity measures from fast marching tractography reveal heritability of white matter architecture. ISBI; Rotterdam, The Netherlands. April 14-17, 2010; 2010. [4 pages; peer-reviewed paper]

10•. Chiang MC, Barysheva M, Lee AD, et al. Genetics of brain fiber architecture and intelligence. J Neurosci 2009;29:2212-2224. This study shows that fractional anisotropy - a commonly used measure of white matter fiber integrity in the brain - is highly heritable and associated with intelligence quotient. [PubMed: 19228974]

11. Posthuma D, De Geus EJ, Baaré WF, et al. The association between brain volume and intelligence is of genetic origin. Nat Neurosci 2002;5:83-84. [PubMed: 11818967]

12. Wallace GL, Lee NR, Prom-Wormley EC, et al. A bivariate twin study of regional brain volumes and verbal and nonverbal intellectual skills during childhood and adolescence. Behav Genet 2010;40:125-134. [PubMed: 20112131]

13. Dufour F, Schaer M, Debbané M, et al. Cingulate gyral reductions are related to low executive functioning and psychotic symptoms in 22q 11.2 deletion syndrome. Neuropsychologia 2008;46:2986-2992. [PubMed: 18616958]

14. Bearden CE, van Erp TGM, Thompson PM, et al. Cortical mapping of genotype-phenotype relationships in schizophrenia. Hum Brain Mapp 2007;28:519-532. [PubMed: 17437284]

15. Lightbody AA, Reiss AL. Gene, brain, and behavior relationships in fragile X syndrome: evidence from neuroimaging studies. Dev Disabil Res Rev 2009;15:343-352. [PubMed: 20014368]

16. Lee $\mathrm{AD}$, Leow $\mathrm{AD}, \mathrm{Lu} \mathrm{A}$, et al. 3D pattern of brain abnormalities in fragile $\mathrm{X}$ syndrome visualized using tensor-based morphometry. NeuroImage 2007;34:924-938. [PubMed: 17161622]

17. Thompson PM, Lee AD, Dutton RA, et al. Abnormal cortical complexity and thickness profiles mapped in Williams syndrome. J Neurosci 2005;25:4146-4158. [PubMed: 15843618]

18. Van Essen DC, Dierker D, Snyder AZ, et al. Symmetry of cortical folding abnormalities in Williams syndrome revealed by surface-based analyses. J Neurosci 2006;26:5470-5483. [PubMed: 16707799]

19. Marenco S, Radulescu E. Imaging genetics of structural brain connectivity and neural integrity markers. Neuroimage. 2009 [Epub ahead of print].

20. Peper JS, Brouwer RM, Boomsma DI, et al. Genetic influences on human brain structure: a review of brain imaging studies in twins. Hum Brain Mapp 2007;28:464-473. [PubMed: 17415783]

21. Schmitt JE, Wallace GL, Lenroot RK, et al. A twin study of intracerebral volumetric relationships. Behav Genet 2010;40:114-124. [PubMed: 20112130]

22. Kremen WS, Prom-Wormley E, Panizzon MS, et al. Genetic and environmental influences on the size of specific brain regions in midlife: the VETSA MRI study. Neuroimage 2010;49:1213-1223. [PubMed: 19786105]

23•. Gilmore JH, Schmitt JE, Knickmeyer RC, et al. Genetic and environmental contributions to neonatal brain structure: a twin study. Hum Brain Mapp. 2010 [Epub ahead of print]. This twin MRI study shows high heritability for brain structure measures, even in infants. This is the youngest twin cohort ever examined with imaging, overcoming the known challenges of scanning infants.

24. Rimol LM, Panizzon MS, Fennema-Notestine C, et al. Cortical thickness is influenced by regionally specific genetic factors. Biol Psychiatry 2010;67:493-499. [PubMed: 19963208]

25. Panizzon MS, Fennema-Notestine C, Eyler LT, et al. Distinct genetic influences on cortical surface area and cortical thickness. Cereb Cortex 2009;19:2728-2735. [PubMed: 19299253]

26. Winkler AM, Kochunov P, Blangero J, et al. Cortical thickness or grey matter volume? The importance of selecting the phenotype for imaging genetics studies. Neuroimage. 2009 [Epub ahead of print]. 
27. Schmitt JE, Lenroot RK, Wallace GL, et al. Identification of genetically mediated cortical networks: a multivariate study of pediatric twins and siblings. Cereb Cortex 2008;18:1737-1747. [PubMed: 18234689]

28. Schmitt JE, Lenroot RK, Ordaz SE, et al. Variance decomposition of MRI-based covariance maps using genetically informative samples and structural equation modeling. Neuroimage 2009;47:5664. [PubMed: 18672072]

29. Toro R, Chupin M, Garnero L, et al. Brain volumes and Val66Met polymorphism of the BDNF gene: local or global effects? Brain Struct Funct 2009;213:501-509. [PubMed: 19205731]

30. Neale, MC.; Cardon, LR. Methodology for genetic studies of twins and families. Dordrecht, the Netherlands: Kluwer Academic Publishers; 1992.

31. Almasy L, Blangero J. Multipoint quantitative-trait linkage analysis in general pedigrees. Am J Hum Genet 1998;62:1198-1211. [PubMed: 9545414]

32. Thompson PM, Cannon TD, Narr KL, et al. Genetic influences on brain structure. Nature Neurosci 2001;4:1253-1258. [PubMed: 11694885]

33. Pietiläinen OPH, Paunio T, Loukola A, et al. Association of AKT1 with verbal learning, verbal memory and regional cortical grey matter density in twins. Am J Med Genet B Neuropsychiatr Genet 2009;150B:683-692. [PubMed: 19051289]

34. Hulshoff Pol HE, Schnack HG, Posthuma D, et al. Genetic contributions to human brain morphology and intelligence. J Neurosci 2006;26:10235-10242. [PubMed: 17021179]

35. Brun CC, Leporé N, Pennec X, et al. Mapping the regional influence of genetics on brain structure variability: a tensor-based morphometry study. NeuroImage 2009;48:37-49. [PubMed: 19446645]

36. Lenroot RK, Schmitt JE, Ordaz SJ, et al. Differences in genetic and environmental influences on the human cerebral cortex associated with development during childhood and adolescence. Hum Brain Mapp 2009;30:163-174. [PubMed: 18041741]

37. McClearn GE, Johansson B, Berg S, et al. Substantial genetic influence on cognitive abilities in twins 80 or more years old. Science 1997;276:1560-1563. [PubMed: 9171059]

38. Haworth CM, Wright MJ, Luciano M, et al. The heritability of general cognitive ability increases linearly from childhood to young adulthood. Mol Psychiatry. 2009 [Epub ahead of print].

39. Pievani M, Rasser PE, Galluzzi S, et al. Mapping the effect of APOE4 on grey matter loss in Alzheimer's disease in vivo. NeuroImage 2009;45:1090-1098. [PubMed: 19349226]

40. Schuff N, Woerner N, Boreta L, et al. MRI of hippocampal volume loss in early Alzheimer's disease in relation to ApoE genotype and biomarkers. Brain 2009;132:1067-1077. [PubMed: 19251758]

41. Hua X, Leow AD, Parikshak N, et al. Tensor-based morphometry as a neuroimaging biomarker for Alzheimer's disease: an MRI study of $676 \mathrm{AD}, \mathrm{MCI}$, and normal subjects. NeuroImage 2008;43:458-469. [PubMed: 18691658]

42. Reiman EM, Chen K, Liu X, et al. Fibrillar amyloid-beta burden in cognitively normal people at 3 levels of genetic risk for Alzheimer's disease. Proc Natl Acad Sci U S A 2009;106:6820-6825. [PubMed: 19346482]

43. Bookheimer S, Burggren A. APOE-4 genotype and neurophysiological vulnerability to Alzheimer's and cognitive aging. Annu Rev Clin Psychol 2009;5:343-362. [PubMed: 19327032]

44. Shaw P, Lerch JP, Pruessner JC, et al. Cortical morphology in children and adolescents with different apolipoprotein E gene polymorphisms: an observational study. Lancet Neurol 2007;6:494-500. [PubMed: 17509484]

45. Filippini N, Zarei M, Beckmann CF, et al. Regional atrophy of transcallosal prefrontal connections in cognitively normal APOE epsilon4 carriers. J Magn Reson Imaging 2009;29:1021-1026. [PubMed: 19388128]

46. Pezawas L, Verchinski BA, Mattay VS, et al. The brain-derived neurotrophic factor val66met polymorphism and variation in human cortical morphology. J Neurosci 2004;24:10099-10102. [PubMed: 15537879]

47. Chiang, MC.; Avedissian, C.; Barysheva, M., et al. Extending genetic linkage analysis to diffusion tensor images to map single gene effects on brain fiber architecture, medical image computing and computer assisted intervention (MICCAI2009); London, UK. Sept. 2009; [8 pages] 
48. Shaw P, Wallace GL, Addington A, et al. Effects of the Val158Met catechol-O-methyltransferase polymorphism on cortical structure in children and adolescents. Mol Psychiatry 2009;14:348-349. [PubMed: 19308019]

49. Ehrlich S, Morrow EM, Roffman JL, et al. The COMT Val108/158Met polymorphism and medial temporal lobe volumetry in patients with schizophrenia and healthy adults. Neuroimage. 2009 [Epub ahead of print].

50. Elvevåg B, Weinberger DR. Introduction: genes, cognition and neuro-psychiatry. Cogn Neuropsychiatry 2009;14 (4-5):261-276. [PubMed: 19634030]

51. Thomason ME, Dougherty RF, Colich NL, et al. COMT genotype affects prefrontal white matter pathways in children and adolescents. Neuroimage. 2010 [Epub ahead of print].

52. Bédard AC, Schulz KP, Cook EH Jr, et al. Dopamine transporter gene variation modulates activation of striatum in youth with ADHD. Neuroimage. 2009 [Epub ahead of print].

53. Blasi G, Lo Bianco L, Taurisano P, et al. Functional variation of the dopamine D2 receptor gene is associated with emotional control as well as brain activity and connectivity during emotion processing in humans. J Neurosci 2009;29:14812-14819. [PubMed: 19940176]

54. Hariri AR, Mattay VS, Tessitore A, et al. Serotonin transporter genetic variation and the response of the human amygdala. Science 2002;297:400-403. [PubMed: 12130784]

55. Ho AJ, Stein JL, Hua X, et al. A commonly carried allele of the obesity-related FTO gene is associated with reduced brain volume in the healthy elderly. Proc Natl Acad Sci U S A 2010;107:8404-8409. This study finds that the FTO gene, which almost a half of Caucasians carry, is associated with brain deficits identifiable on MRI. The gene also confers an increased risk for obesity. [PubMed: 20404173]

56. Raji CA, Ho AJ, Parikshak N, et al. Brain structure and obesity. Hum Brain Mapp. 2009 [Epub ahead of print].

57. Stein JL, Hua X, Morra JH, et al. Genome-wide association study of temporal lobe structure identifies novel quantitative trait loci for neurodegeneration in Alzheimer's disease. Neuroimage 2010;51:542-554. [PubMed: 20197096]

58. Purcell S, Neale B, Todd-Brown K, et al. PLINK: a tool set for whole-genome association and population-based linkage analyses. Am J Hum Genet 2007;81:559-575. [PubMed: 17701901]

59. Parsons CG, Stoffler A, Danysz W. Memantine: a NMDA receptor antagonist that improves memory by restoration of homeostasis in the glutamatergic system: too little activation is bad, too much is even worse. Neuropharmacology 2007;53:699-723. [PubMed: 17904591]

60•. Dickson SP, Wang K, Krantz I, et al. Rare variants create synthetic genome-wide associations. PLoS Biol 2010;8:e1000294. This provocative study argues that rare variants may be responsible for some of the results seen in GWA studies. It advocates deeper sequencing of the genome than is customary in GWA scanning studies. [PubMed: 20126254]

61. Choi M, Scholl UI, Ji W, et al. Genetic diagnosis by whole exome capture and massively parallel DNA sequencing. Proc Natl Acad Sci U S A 2009;106:19096-19101. [PubMed: 19861545]

62. Meda SA, Jagannathan K, Gelernter J, et al. A pilot multivariate parallel ICA study to investigate differential linkage between neural networks and genetic profiles in schizophrenia. Neuroimage. 2009 [Epub ahead of print].

63. Lee, AD.; Lepore, N.; de Leeuw, J., et al. Multivariate variance-components analysis in DTI. ISBI; Rotterdam, The Netherlands. April 14-17, 2010; 2010. [4 pages; peer-reviewed paper]

64. Langers DR, Jansen JF, Backes WH. Enhanced signal detection in neuroimaging by means of regional control of the global false discovery rate. Neuroimage 2007;38:43-56. [PubMed: 17825583]

65. Chiang, MC.; Barysheva, M.; Toga, AW., et al. BDNF effects on brain fiber microstructure replicated in two twin samples $(\mathrm{N}=455)$. Organization for Human Brain Mapping; Barcelona, Spain: 2010.

66. Wray NR, Goddard ME, Visscher PM. Prediction of individual genetic risk to disease from genome-wide association studies. Genome Res 2007;17:1520-1528. [PubMed: 17785532]

67. Bertolino A, Fazio L, Di Giorgio A, et al. Genetically determined interaction between the dopamine transporter and the D2 receptor on prefrontostriatal activity and volume in humans. J Neurosci 2009;29:1224-1234. [PubMed: 19176830] 
68. Pezawas L, Meyer-Lindenberg A, Goldman AL, et al. Evidence of biologic epistasis between BDNF and SLC6A4 and implications for depression. Mol Psychiatry 2008;13:709-716. [PubMed: 18347599]

69. Bigos KL, Weinberger DR. Imaging genetics: days of future past. Neuroimage. 2010 [Epub ahead of print].

70. McCarthy MI, Abecasis GR, Cardon LR, et al. Genome-wide association studies for complex traits: consensus, uncertainty and challenges. Nat Rev Genet 2008;9:356-369. [PubMed: 18398418]

71 - Joyner AH, Roddey JC, Bloss CS, et al. A common MECP2 haplotype associates with reduced cortical surface area in humans in two independent populations. Proc Natl Acad Sci U S A 2009;106:15483-15488. This study is notable as one of the few studies (at the time of writing) that have used two independent samples to replicate an association effect. [PubMed: 19717458]

72••. Cichon S, Craddock N, Daly M, et al. Genomewide association studies: history, rationale, and prospects for psychiatric disorders. Am J Psychiatry 2009;166:540-556. This study by the Psychiatric Genetics Consortium shows the need for, and value of, multisite collaborations on GWA studies. [PubMed: 19339359]

73. Jack CR Jr, Bernstein MA, Borowski BJ, et al. Update on the MRI core of the Alzheimer's disease neuroimaging initiative. J Alzheimer's Dement 2010;6:212-220.

74. Rasch B, Papassotiropoulos A, de Quervain DF. Imaging genetics of cognitive functions: focus on episodic memory. Neuroimage. 2010 [Epub ahead of print]. 


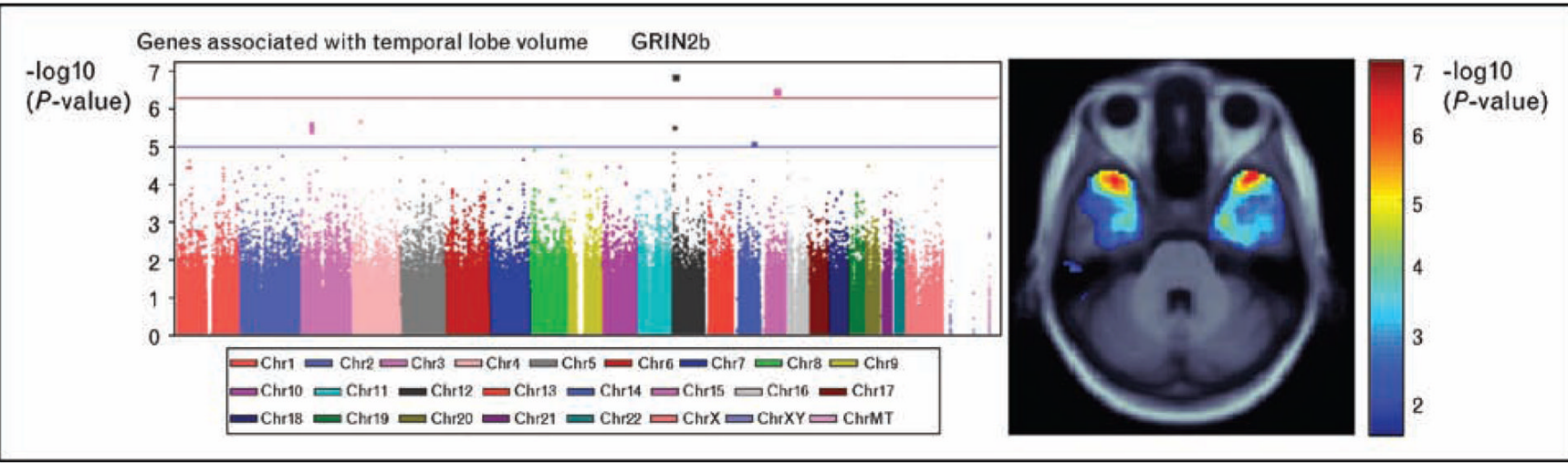

Figure 1. Genome-wide association study of temporal lobe structure

The GWA study identified a common glutamate receptor variant (GRIN2b) with suggestive evidence of association with temporal lobe volume and increased risk for Alzheimer's disease in 740 brain MRI scans. The plot on the left is a standard output from the PLINK software [58] - it shows the significance of association between the image-derived measure of interest (here temporal lobe volume) and variants at each of 600000 locations on the genome. By performing genetic association to assess the effects of variants in this SNP at millions of points in a brain image, associations were detected and mapped in 3D (right). Adapted from [57]. 